\title{
Insight of microRNA role in Colorectal Cancer
}

\author{
Kwan-Liang Lye ${ }^{1 *}$, Loh Teng-Hern Tan ${ }^{2}$, Hui-Min Yap ${ }^{1}$ \\ ${ }^{1}$ Department of Biomedical Sciences Faculty of Medicine and Health Sciences, Universiti Putra Malaysia, 43400 UPM \\ Serdang, Selangor Darul Ehsan, Malaysia \\ ${ }^{2}$ Novel Bacteria and Drug Discovery Research Group (NBDD), Microbiome and Bioresource Research Strength (MBRS), \\ Jeffrey Cheah School of Medicine and Health Sciences, Monash University Malaysia, 47500 Bandar Sunway, Selangor \\ Darul Ehsan, Malaysia
}

\begin{abstract}
The colorectal cancer is among the most predominant cancer in the world including Malaysia. Numerous factors could contribute towards colorectal carcinogenesis and one of the factors is genetic predisposition. Mutations in the V-KiRas2 (Kras) oncogene have been implicated in 30-50\% of the colorectal cancer patients and usually lead to poorer prognosis. The challenging ability for the early detection of colorectal cancer still poses an enormous challenge to oncologist as there are limited or no signs or symptoms in the early stage of colorectal cancer. Many studies were conducted hoping to further understand colorectal cancer for a better diagnosis and prognosis. As early detection of colorectal cancer frequently leads to good prognosis. The gold standard for prognosis depends on the stage of the tumor at the time of diagnosis. Lately a group of small, non-coding RNAs termed microRNAs (miRNAs) exhibited capable outcomes in cancer research. Numerous miRNAs were discovered to play a key role in regulatory mechanism in numerous cancers. Differential miRNAs expression among tumors and non-tumor controls are highly valuable in recognizing miRNAs that could have vital role in carcinogenesis. Recently some miRNAs were discovered to play a vital role in colorectal carcinogenesis. Thus, miRNAs have emerged as highly useful tool for scientists to comprehend carcinogenesis better. For example, miR-21 and miR-106a were highly expressed in colorectal cancer. While miRNAs including miR-17-92 cluster, miR-21, miR-34, miR-135 and miR-196a also exhibited high association with colorectal cancer. Therefore, this article aims to provide insight of miRNAs role in colorectal cancer for a better understanding of this disease.
\end{abstract}

Keywords: microRNA; miRNA; colorectal cancer; Malaysia; cancer

Received: $9^{\text {th }}$ April 2020

Accepted: $10^{\text {th }}$ May 2020

Published Online: $15^{\text {th }}$ May 2020
*Correspondence: Kwan-Liang Lye, Department of Biomedical Sciences Faculty of Medicine and Health Sciences, Universiti Putra Malaysia, 43400 UPM Serdang, Selangor Darul Ehsan, Malaysia; kl_lye86@hotmail.com

Citation: Lye KL, Tan L-HT and Yap H-M. Insight of microRNA role in Colorectal Cancer. Prog Microbes Mol Biol 2020; 3(1): a0000083. https://doi.org/10.3687/pmmb.a0000083

\section{INTRODUCTION}

\section{Colorectal Cancer}

Colorectal cancer is a kind of cancer that forms in the tissues of the colon and rectum. Classically, most colorectal cancers are adenocarcinomas or cancer arises from cells making and releasing fluids such as mucus. Alike other cancers ${ }^{[1]}$, colorectal cancer is a multifactorial disease that exhibited complex interactions among inherited susceptibility and environmental factors leading towards the development of the disease $\mathrm{e}^{[2,3]}$.

\section{Prevalence of colorectal cancer}

A total of 115,238 new cancer cases were diagnosed in Malaysia for the period of 2012-2016. The Malaysian National Cancer Registry Report 2012-2016 indicated that age-standardized incidence rates (ASR) for all cancers were 86.1 for males and 101.6 for females per 100,000 populations ${ }^{[3]}$. The 5 most common cancers among Malaysian were reported as breast, colorectal, trachea, bronchus and lung (TBL), lymphoma and nasopharynx cancer. The statistics indicated that colorectal cancer ranked 2nd most common cancer in Malaysia. Total ASR was at 13.5 per 100000 population. Colorectal cancer was somewhat higher in males (14.8 per 100000) as compared to females (11.1 per 100000) ${ }^{[3]}$. Looking at different ethnicities, Chinese documented the highest ASR (21.4 per 100000), followed by Indian (11.3 per $100000)$ and Malay $(9.5 \text { per } 100000)^{[4]}$. Data obtained from the National Cancer Registry 2007 indicated that colorectal cancer ranked $2 \mathrm{nd}$ as one of the most common cancers in Malaysia, with ASR for male at 85.1 per 100000 and female at 94.4 per 100000 population ${ }^{[5]}$.

\section{Diagnosis of colorectal cancer}


Diagnosis is extremely important in the management of colorectal cancer. Currently there are few types of tests and procedures used to diagnose and detect colorectal cancer as listed here:

- Fecal occult blood test — detecting occult blood in the feces

- Double contrast barium enema - liquid containing barium is placed into the rectum and coats the lower gastrointestinal tract. X-rays are undertaken to detect abnormal regions of the colorectal

- Colonoscopy - a colonoscope is introduced via the rectum into the colon to investigate polyps, abnormal areas or cancer

- $\quad$ Sigmoidoscopy - a sigmoidoscope is introduced via the rectum into the sigmoid colon to view for abnormal areas, polyps or cancer.

- Virtual colonoscopy - using computed tomography to generate series of photos of the colon and allows the view of any abnormal areas such as polyps in the colon.

\section{Staging of colorectal cancer}

Staging is the important procedure of investigating how extensive a cancer has spread and is one of the most important factors in defining the prognosis and treatment choices for cancer patients. Several staging systems are employed for colorectal cancer. The most common staging system is the TNM system established by American Joint Committee on Cancer (AJCC). TNM system illustrates three key evidence:

- T illustrates how extensive the main tumor has spread into the wall of the intestine.

- $\quad \mathbf{N}$ explains the extent of spread to nearby (regional) lymph nodes.

- M specifies whether the cancer has metastases or spread to the other organs of the body.

The wider staging of a cancer is usually represented by roman numbering for instance I, II, III and IV originated from the TNM values. Specifics of this staging system are demonstrated in Table 1.

Table 1. Colorectal cancer staging system.

\begin{tabular}{|l|l|l|}
\hline AJCC stage & TNM stage & Details \\
\hline 0 & Tis N0 M0 & Tumor has not expanded beyond the inner layer (mucosa) \\
\hline I & T1 N0 M0 & Tumor has invaded submucosa \\
\hline I & T2 N0 M0 & Tumor has invaded muscularis propria \\
\hline II A & T3 N0 M0 & Tumor has invaded subserosa \\
\hline II B & T4 N0 M0 & Tumor has invaded adjacent organs or perforated the visceral peritoneum \\
\hline III A & T1-2 N1 M0 & Tumor has extended to 1-3 regional lymph nodes. T1 or T2. \\
\hline III B & T3-4 N1 M0 & Tumor has extended to 1-3 regional lymph nodes. T3 or T4. \\
\hline III C & T1-4 N2 M0 & Tumor has extended to 4 or more regional lymph nodes. \\
\hline IV & T1-4 N0-2 M1 & Tumor has extended to 1 or more distant organ(s) or set of lymph nodes. \\
\hline
\end{tabular}

Another staging system utilized is the Dukes system, it is a less complex staging system. Itemized here are the descriptions of the stages in the Dukes system:

- A denotes the tumor that is confined to the intestinal wall.

- B denotes the tumor that begins invading over the intestinal wall.

- C denotes the involvement of lymph node(s)

- D denotes distant metastases

\section{Present treatment for colorectal cancer}

Different kinds of treatments are available for colorectal cancer and are administered according to the diagnosis of the colorectal cancer by oncologist. The available treatments for colorectal cancer are discussed below:

- Surgery - as one of the most frequent treatment for colorectal cancer. A clinician could do local excision if the cancer is still at an early stage. If the cancer is larger, the clinician could do colectomy to remove the cancer and nearby normal tissues. Additional options consist of radiofrequency ablation and cryotherapy.

- Chemotherapy - to use of medications that destroys cancer cells or inhibiting cancer cells division.
Numerous medications could be given concurrently to increase treatment effectiveness. One of the regular medicines for treating colorectal cancer is 5-Fluorouracil (5-FU), commonly apply concurrently with oxaliplatin and leucovorin in a combination recognized as FOLFOX.

- Radiotherapy - the use of high doses of radiation to kill cancer cells via destructing the targeted cells genetic materials. There are 2 types of radiotherapy, the external radiation therapy that uses a beam of radiation to target on the cancer area and repeated over a few days. The other would be the internal radiation therapy that uses radioactive materials inserted into or near the tumor via small thin tubes or needle.

- Immunotherapy - the use of individual body's immune system to combat cancer. This therapy primarily promotes the immune system to response and combat more effectively against cancer. Monoclonal antibodies therapy is one of the immunotherapies using generated monoclonal antibodies to target the tumor in the body and to deliver drug or radioactive materials directly to tumor cells. Other examples of immunotherapy are colony-stimulating factors, tumor vaccines and biological response modifiers.

Other than existing treatment options, scientists conducted many experiments in hope to search for useful metabolites 
or compounds that could potentially inhibit the growth of cancer cells ${ }^{[6-12]}$ especially colorectal cancer ${ }^{[13-19]}$. Researches have gained some good findings, but more tests need to be done before these potential compounds could be ready for clinical application.

\section{THE RAS ONCOGENE}

The Ras is a family of related proteins known as small GTPase. They are vital for signal transmission between cells. The naming of "Ras" derived from "Rat sarcoma", indicating the source from which the first member of the protein family was discovered. The ras family comprises of 3 members, Nras, Hras and $\mathrm{Kras}^{[20]}$. Members of the ras family are triggered after a nearby transmembrane receptor is bound by its corresponding ligand. The ras protein is activated by guanine nucleotide exchange factors (GEFs) that leads to the development of GTPbound state, followed by inactivation by GTPase activating proteins (GAPs) forming the GDP-bound state by GTP hydrolysis. Consequently, these will be activated and regulating other genes participating in cell differentiation, growth and survival. Hence, mutation in ras genes could lead to overexcited ras signaling, therefore triggering uncontrolled cell division and cell growth that would eventually become cancerous ${ }^{[21]}$.

\section{Kras mutation in colorectal cancer}

Kras gene is a vital gene in colorectal cancer, this gene expresses a protein involved in the epidermal growth factor receptor (EGFR) signaling pathway. The Kirsten rat sarcoma viral oncogene homolog or Kras gene belongs to the Ras family of oncogenes, and mutations are common notably in colorectal cancer, pancreatic cancer and lung cancer. Kras gene mutation is among the earlier events in colorectal carcinogenesis, and the ability to detect Kras gene mutation is very important for diagnosis. Kras mutation was reported in the earlier stages of molecular alteration contributing to the development of colorectal adenoma to carcinoma. The Kras protein has vital role in tumor growth via the regulation of downstream proteins involved in survival, proliferation, and metastasis ${ }^{[21,22]}$.

The prevalence rate of Kras mutation was reported to be $20 \%$ to $50 \%{ }^{[23,24]}$. Reported here are countries and their respective rate of Kras mutation amongst colorectal cancer patients; Italy (46.3\%), USA (40\%), Iran (37.4\%), Turkey (34.2\%), Jordan (33.3\%), Taiwan (26.5\%) and Egypt $(18.4 \%)^{[25,26,27]}$. One of the most common Kras mutation occurs at codon 12 and codon $13^{[28]}$. It was reported that codon 12 has higher mutation rate as compared to codon

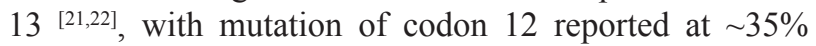
based by Bazan et al. ${ }^{[29]}$. Researchers suggested the high mutation rate of codon 12 is because of the vulnerability of codon 12 to carcinogen binding, furthermore along with the poor repair mechanism of the resulting adduct ${ }^{[21,22]}$. Also, other studies indicated that Kras mutation normally implicated codon $12,13,59$ or $61^{[30,31]}$.

Reports indicated that Kras mutations contributed to higher probability of death and lower progression-free survival ${ }^{[22]}$. Reinacher-Schick et al. ${ }^{[32]}$ also showed that Kras mutations associated to lower progression-free survival in patients with advanced colorectal cancer treated with oxaliplatin chemotherapy. The increase of response rate associates with increased progression free survival in colorectal cancer. Nevertheless, Kras mutations have been connected to decreased response degree towards chemotherapeutic agents. Lievre et al. ${ }^{[33]}$ reported that Kras mutation was decreasing the reaction of anti-epidermal growth factor receptor (EGFR), therefore patients with mutant Kras demonstrated lower survival rate as compared to patients with wild-type Kras. Hence, the prognosis of patients could be improved by verifying the mutation status of the Kras gene.

\section{THE MicroRNAs}

MicroRNAs (miRNAs) are small, non-coding RNA found in the genomes of vertebrates, invertebrates and plants ${ }^{[34]}$. The typical size of mature miRNAs is 21-25 nucleotides. MiRNAs demonstrated important role in many vital processes for instance cell proliferation, differentiation and apoptosis ${ }^{[35]}$. MiRNAs controls gene expression by various ways for example mRNA cleavage, deadenylation and translational repression. The exciting element is that miRNA is able to control the expression level by partial complementary binding to the target mRNA. This allows a single miRNA to control and regulate more than one target mRNA and perform several roles in our biological processes.

\section{Discovery of miRNAs}

The first miRNA, the lin-4 was discovered in 1993 by the Ambros's and Ruvkun's research team ${ }^{[36,37]}$. The gene lin4 was detected by isolation of null mutation that triggers a breakdown in temporal growth in Caenorhabditis elegans ${ }^{[36]}$. Ambros et al. ${ }^{[34]}$ reported a $700 \mathrm{bp}$ fragment that may well have lin- 4 gene but unable to locate the conventional start and stop codons, hence indicated that lin-4 is not encoding protein. Researchers also discovered two small transcripts of lin-4 of $61 \mathrm{nt}$ and $22 \mathrm{nt}$ in length that correspond to the common precursor miRNA and mature miRNA length ${ }^{[37]}$.

The second miRNA discovered was let-7, that is likewise a heterochronic gene of C. elegans. Reinhart et al. ${ }^{[39]}$ discovered that let-7 was a $21 \mathrm{nt}$ RNA regulating the transition stage from L4 to adult in the larval development. Dissimilar to the lin-4, let-7 sequence is conserved across species from invertebrates to complex organisms for instance humans. Nevertheless, let-7 was not found in unicellular organisms and plants. Curiously, the expression level of let-7 is dissimilar in different types of human tissue ${ }^{[40]}$. The finding that let- 7 was conserved across numerous species initiated the surge of research in the small RNA called microRNA (miRNA). Till date, more than 38 thousand miRNAs is listed in the miRBase database (http:// http://www.mirbase. org/) and keeps rising.

\section{miRNA Synthesis}

As depicted in Figure 1, miRNAs synthesis starts in the nucleus, with the primary transcripts (pri-miRNAs) processed into miRNA precursor (pre-miRNA) facilitated by Drosha and Dicer, which are RNase III enzymes. The pre-miRNAs are next exported from the nucleus into the cytoplasm by Exportin-5 and cut by Dicer into a 22-nucleotide mature double stranded miRNA ${ }^{[41]}$. This strand is then fused into the Argonaute protein to create the effector RNA-induced 
silencing complex (RISC) after which the miRNA and its mRNA target interact. The miRNA will only interact with mRNAs containing anti-sense sequences. Nevertheless, this interaction could occur even if they are partially complementary to each other ${ }^{[42]}$.

\section{miRNA Role in Human Diseases}

The discovery of miRNAs had quickly led to key research conducted to investigate their roles in humans especially in disease progression. Cancers were extensively studied, as one of the most common chronic disease affecting human. Furthermore, miRNAs were as well correlated to a multitude of other diseases. Van Rooij et $a{ }^{\left[{ }^{[43]}\right.}$ firstly reported the association of miRNAs with cardiac hypertrophy and heart failure. The study utilized miRNA microarray analysis demonstrated 12 miRNAs were deregulated during cardiac hypertrophy and heart failure $^{[43]}$. Furthermore Tijsen et al. ${ }^{[44]}$ reported another miRNA, miR-423-5p was elevated in heart failure patients. While for acute myocardial infarction patients, miR-208b and miR-499 was discovered to be greatly elevated and associated with the plasma troponin level ${ }^{[45]}$.

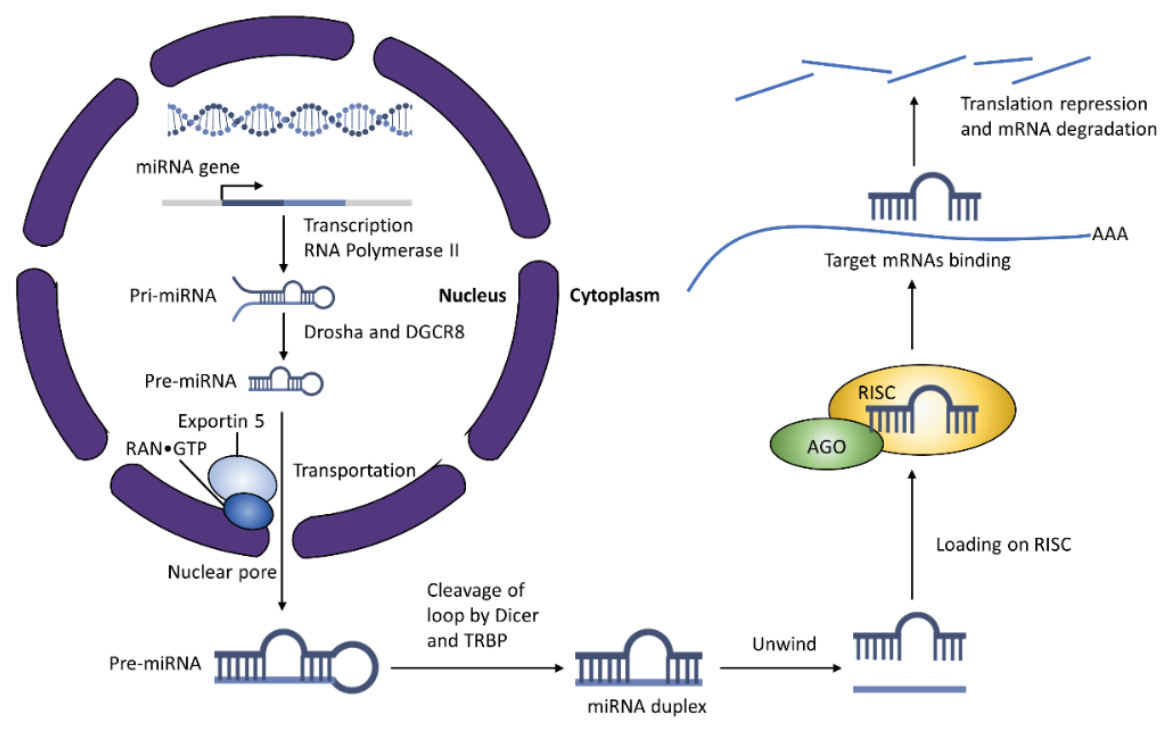

Figure 1. The miRNA synthesis pathway.

'Moreover, miRNAs have demonstrated important role in autoimmune diseases. Stanczyk et al. ${ }^{[46]}$ demonstrated miRNAs role in autoimmune diseases with the discovery of miR-146 and miR-155 overexpressed in rheumatoid arthritis synovial fibroblast and synovial tissue. The finding agreed with another study by Nakasa et al. ${ }^{[47]}$ that validated the expression of miR-146 in rheumatoid arthritis synovial tissue. In 2007, Dai et al. ${ }^{[48]}$ described a total of 16 miRNAs were differentially expressed in patients of systemic lupus erythematosus (SLE). Both the miR-21 and miR-148a were overexpressed in CD4+ T cells of SLE patients and contributing to down-regulation of the DNMT1 gene, triggering DNA hypomethylation ${ }^{[49]}$.

Acouple ofmiRNAs were correlated to neurodegenerative diseases for instance Parkinson, Alzheimer and Huntington's disease. Lukiw (2007) ${ }^{[50]}$ reported that in Alzheimer's patients, miR-9, miR-25b and miR128 were up-regulated, whereas miR-124a was downregulated. An increase in miR-9, miR-128 and miR-125b were observed on cultured human fetal brain-derived primary neural cells, that was treated with metal salts to create reactive oxygen species (ROS) ${ }^{[51]}$. While miR$133 \mathrm{~b}$ was absent in brain tissue of Parkinson's disease patients, indicating miR-133b vital roles for maturation and function of dopamigernic neurons ${ }^{[52]}$.

\section{miRNA in cancer}

In 2002, miRNAs were first reported to be involved in cancer, with miR-15 and miR-16 discovered to be downregulated or deleted in chronic lymphocytic leukemia $(\mathrm{CLL})^{[53]}$. Cimmino et al. ${ }^{[54]}$ demonstrated that miR-15 and miR-16 have a role in apoptosis by targeting Bcl2 mRNA. Various studies then reported different miRNA expression in almost all types of cancer. MiRNAs can behave as tumor suppressors or oncomiRs in carcinogenesis ${ }^{[5,56,57,58]}$. MiRNAs that were up-regulated act as oncomiRs, while down-regulated miRNAs in cancer usually operate as tumor suppressor. The overexpressed miRNAs leading to cancerous growth operate as oncogenes, whereas underexpressed miRNAs leading to cancerous growth act as tumor suppressor. There were numerous miRNAs identified as tumor suppressor. Johnson et al. ${ }^{[59]}$ demonstrated that let-7 expression was down-regulated in lung cancer tissue as compared to normal tissue. Furthermore, it associates with the increased of Ras protein in the lung cancer samples. While study by Garzon et al. ${ }^{[60]}$ indicated that miR-29 was down-regulated in acute myeloid leukemia. While miR-34 was reported to be down-regulated in colon, pancreatic and breast cancers ${ }^{[61]}$. The miR-155 was among the first miRNA to be linked as oncomiRs. It was up-regulated in various cancers such as acute myeloid leukemia ${ }^{[60]}$, Hodgkin disease ${ }^{[62]}$, Burkitt lymphoma ${ }^{[63]}$, and lung cancer ${ }^{[64]}$.

\section{miRNAs in colorectal cancer}

MicroRNAs (miRNAs) have exhibited vital role in colorectal cancer genesis, progression and response to 
treatments. Researchers demonstrated that in colorectal cancer samples, more miRNAs were up-regulated than down-regulated ${ }^{[65,66,67]}$. The miR-21 is one of the properly studied miRNA and discovered to be associated in many types of cancers. It was reported among the first miRNA discovered as oncomiR and was associated to a multitude of tumor suppressor genes for instance PDCD4, PTEN and $B C L-2$. Faltejskova et al. ${ }^{[68]}$ indicated higher expression of miR-21 was associated with shorter general survival of colorectal cancer patients. They performed silencing of miR-21 expression in DLD1 cell lines and observed $30 \%$ suppression of the cancer cells migration ability, thus leading to lower cancer cells viability. This finding demonstrated the role of miR-21 in cancer cells migration in tumorigenesis. Likewise, Link et al. ${ }^{[69]}$ demonstrated that miR-21 was highly expressed in patients with adenomas and colorectal cancer as compared to healthy individuals. Another study of stage II colon cancer patient exhibited increased miR-21 expression levels were associated to decrease recurrence-free cancer-specific survival ${ }^{[70]}$. Furthermore, the plasma miR-21 was able to differentiate colorectal cancer patients from normal controls with $90 \%$ sensitivity and specificity ${ }^{[71]}$. These findings demonstrated that miR-21 could be a reliable and non-invasive marker for colorectal cancer.

Link et al. ${ }^{[69]}$ reported that miR-106a was up-regulated in colorectal cancer patients when compared with normal patients. Furthermore miR-106a was overexpressed in colorectal cancer and regulates the retinoblastoma $1(R B 1)$ gene in sporadic colorectal cancer patients ${ }^{[72]}$. Lately, Feng et al. ${ }^{[73]}$ demonstrated that miR-106a was highly expressed in metastatic colorectal cancer cells and regulating the migration and invasion both in vitro and in vivo. The miR-106a prevents the expression of transforming growth factor-b receptor 2 (TGFBR2), leading to increase tumor cells migration and invasion. While Diaz et al. ${ }^{[74]}$ stated the downregulation of miR-106a contributes to lower disease-free survival and overall survival of colon cancer patients, regardless of the tumor stage.

The miR-135b was regularly found up-regulated in cancer samples. Faltejskova et al. ${ }^{[75]}$, reported the increased of miR-135b expression in CRC tumor tissues. Furthermore, miR-135b was correlated with higher serum levels of CEA and CA19-9. Moreover, Xu et al. ${ }^{[76]}$ indicated the elevated expression levels of miR-135b in CRC tissues compared to normal tissues, and the positive association of miR-135b with the clinical stage. The miR-135b targets the adenomatous polyposis coli (APC) gene, an important gene in colorectal carcinogenesis ${ }^{[7]}$. They discovered that miR-135b was up-regulated in colorectal carcinomas and associates with the low APC mRNA levels leading to colorectal cancer.

The high-throughput sequencing was used to compare between paired tumor and normal tissue, and results identified that 37 miRNAs were dysregulated, with miR1 among the down-regulated miRNAs ${ }^{[77,78]}$. Furthermore, miR-1 down-regulation was correlated to colorectal cancer progression, hence attributes that miR-1 can be a potential tumor suppressor via down-regulating MET oncogene at RNA and protein level ${ }^{[79]}$.

For miR-504, researchers have reported its association in cancer in various studies ${ }^{[80,81,82]}$. The miR-504 was upregulated in oral cancer, by increasing the invasion and migration capabilities of oral cancer cells ${ }^{[80]}$. While $\mathrm{Hu}$ et $a l .{ }^{[81]}$ indicated that miR-504 down-regulates the $\mathrm{p} 53$ protein via binding to the 3'-UTR of p53 gene, hence stimulating tumorigenesis. In 2011, another study also demonstrated that miR-504 down-regulates p53 protein levels and damages its function particularly in p53-mediated apoptosis and G1 cell cycle arrest ${ }^{[82]}$.

Bauer and Hummon (2012) ${ }^{[83]}$ indicated that miR-145 was down-regulated in colon cancer and created distinctive molecular alterations. Earlier studies have indicated that these miRNAs were also down-regulated in more types of cancers. Kent et al. ${ }^{\left[{ }^{[4]}\right.}$ established that Ras activation leads to the down-regulation of miR-145 that propels tumorigenesis. The down-regulation of miR-145 was reported to increase risk of esophageal cancer ${ }^{[85]}$. Whereas in bladder cancer, both miR-133a and miR-145 were discovered to be downregulated and targeted the oncogenic FSCN1 mRNA ${ }^{[86]}$. Hence, miR-133a and miR-145 could act as a probable tumor suppressor by regulating the FSCN1 gene.

Three miRNAs from the miR-182/183 cluster, namely miR-182, miR-183 and miR-96 were up-regulated various studies $^{[78,87,88,89]}$. Sarver et al. ${ }^{[78]}$ indicated that these miRNAs (miR-182, miR-183 and miR-96) were up-regulated in colon cancer tissues. The miR-183 was overexpressed in rhabdomyosarcoma and colon cancer ${ }^{[00]}$, and the miR-183 demonstrated a function as oncomiR via controlling the tumor suppressor EGR1 and PTEN and advocating tumor cell migration ${ }^{[90]}$. Cekaite et al. ${ }^{[87]}$ demonstrated that miR182 was overexpressed by more than 2 fold in colon cancers throughout all clinical stages. Likewise, miR-96 was among the miRNAs reported to be up-regulated in colorectal cancer sample as compared to adjacent normal tissue ${ }^{[78,86]}$. Intriguingly, $\mathrm{Yu}$ et al. ${ }^{[89]}$ reported differing finding of miR96 down-regulated in pancreatic cancer and act as a tumor suppressor gene via inhibiting Kras oncogenic gene.

The miR-224 expression was elevated in colorectal cancer cell lines and in wild type Kras and BRAF colorectal tumors. Arndt et al. ${ }^{\left[{ }^{11]}\right.}$ indicated that miR-224 was overexpressed and correlated to colorectal cancer tumor progression. Yet, Mencia et al. ${ }^{[92]}$ suggested that miR-224 was underexpressed in colorectal cancer cell lines and leading to the cells exhibiting rise in resistance towards methotrexate. Likewise, previous studies have suggested that miR-203 was up-regulated in colorectal cancer, but down-regulated in another study ${ }^{[93]}$. Chiang et al. ${ }^{\left[{ }^{[9]}\right.}$ reported that miR-203 has really low expression in colorectal cancer tissue and cell lines.

MiR-31, a miRNA discovered to be highly expressed in colorectal cancer tissues and correlated with advance tumor stage and poor differentiation ${ }^{[95]}$. Furthermore Wang et al. ${ }^{[96]}$ also reported miR-31 to be up-regulated in CRC samples and positively related to advanced TNM stage. While reports indicated that miR-17 was up-regulated in colorectal cancer and promotes tumor cell proliferation, growth and cell cycle $\operatorname{progression}^{[97]}$.

\section{miRNAs in cancer pathways}

MiRNAs participation in cancer pathways have been 
highlighted in many studies. Many proteins in key signaling pathways of colorectal cancer are changed and controlled by miRNAs. The Wnt pathway is one of the important pathways in early colorectal cancer development. In the Wnt pathway, inactivation of the APC gene is one of the key beginning steps for colorectal carcinogenesis $^{[98]}$. Nagel et al. ${ }^{[77]}$ suggested that miR$135 \mathrm{a}$ and miR-135b reduce the translation of APC gene in vitro. Also, the expression of these miRNAs increases in colorectal cancer and associated with low expression of APC gene.

EGFR and Kras signaling pathways lead to the initiation of numerous signal transduction molecules which started a cascade of downstream effectors regulating tumor growth, angiogenesis and metastasis ${ }^{[99]}$. The upregulation of Kras will start a cascade of downstream activation of MEK gene, RAF gene and MAPK gene, therefore increasing the proliferation of tumor cells ${ }^{[100]}$. The miR-1 and miR-106a were reported for presumed targets of the MAPK gene family, therefore play a part in the Kras signaling pathway. The PI3K pathway is an important signaling pathway downstream of the EGFR pathway. Researchers reported that miR-135b and miR21 targets the genes involved in PI3K pathway. With miR-21 clearly repressing the tumor suppressor, PTEN gene leading to lower survival rate of cancer patients ${ }^{[101]}$.

Another renowned tumor suppressor gene, p53 was mutated in estimated $50-75 \%$ of all colorectal cancer and other tumors. The p53 protein responds to DNA damage and deregulation of oncogenes through the initiation of cell cycle checkpoints, cellular senescence or apoptosis ${ }^{[102,103]}$. The miR-504 exhibited putative target of $B C L-2$ gene, one of the gene that regulates the p53 pathway. Researchers indicated that miR-504 down-regulates the $\mathrm{p} 53$ protein, thus promoting cancer progression $^{[81]}$. These results was also in agreement by Feng et al. ${ }^{[82]}$ that indicated that miR-504 down-regulates p53 protein and damages its function in p53-mediated apoptosis and G1 cell cycle arrest.

\section{CONCLUSION}

This article provided vital insight into the roles of miRNAs in colorectal cancer. In colorectal cancer, studies indicated that many miRNAs are involved in the pathogenesis of the disease. They control the known oncogenes or tumor suppressor pathways by targeting proteins for instance p53, Kras and phophatidylinositol3-kinase (PI3K). Nagel et al. ${ }^{[77]}$ demonstrated that miR-135a and miR-135b reduce the translation of the APC transcript in vitro. The inactivation of the APC gene an important stage in colorectal carcinogenesis. Furthermore, let-7 and miR-143 were described to target Kras oncogene. The Kras signaling leads to the initiation of numerous signal transduction molecules that starts a cascade of downstream effectors regulating angiogenesis, tumorigenesis and metastasis. Some miRNAs were discovered to be correlated with colorectal cancer for instance the miR-17-92 cluster, miR-21, miR-34, miR135 and miR-196a $\mathrm{a}^{[104,105,106,107]}$. In conclusion, this article shed light of miRNAs role in colorectal cancer that enabled a much better understanding of the disease.

\section{Author Contributions}

The literature review and manuscript writing were performed by K-LL, LT-HT and H-MY.

\section{Conflict of Interest}

The authors declare that there is no conflict of interest in this work.

\section{Reference}

1. Chow YP, Mohd Yunos RI, Rose IM, et al. Characterization of somatic mutations in Malaysian luminal breast cancer. Prog Microbes Mol Biol 2018; 1(1): a0000014.

2. Tieng FYF, Baharudin R, Abu N, et al. Single cell transcriptome in colorectal cancer - Current updates on its application in metastasis, chemoresistance and the roles of circulating tumor cells. Front Pharmacol 2020; 11: 135 .

3. Mohd Yunos RI, Ab Mutalib NS, Khor SS, et al. Whole exome sequencing identifies genomic alterations in proximal and distal colorectal cancer. Prog Microb Mol Biol 2019; 1(1): a0000036.

4. Azizah AM, Hashimah B, Nirmal K, et al. Malaysia national cancer registry report 2012-2016. Ministry of Health Malaysia, 2019; Available at: http://nci.moh.gov.my. Accessed May 6, 2020.

5. Jemal A, Bray F, Center MM, et al. Global cancer statistics. CA Cancer J Clin 2010; 61: 69-90.

6. Ser H-L, Tan LT-H, Law JW-F, et al. Focused review: cytotoxic and antioxidant potentials of mangrove-derived Streptomyces. Fron Microbiol 2017; 8: 2065.

7. Ser H-L, Yin W-F, Chan K-G, et al. Antioxidant and cytotoxic potentials of Streptomyces gilvigriseus MUSC $26^{\mathrm{T}}$ isolated from mangrove soil in Malaysia. Prog Microbes Mol Biol 2018; 1(1): a0000002.

8. Law JW-F, Chan K-G, He Y-W, et al. Diversity of Streptomyces spp. from mangrove forest of Sarawak (Malaysia) and screening of their antioxidant and cytotoxic activities. Sci Rep 2019; 9(1): 1-15.

9. Azman AS, Othman I, Fang C-M, et al. Antibacterial, anticancer and neuroprotective activities of rare Actinobacteria from mangrove forest soils. Indian J Microbiol 2017; 57(2): 177-187.

10. Law JW-F, Pusparajah P, Ab Mutalib NS, et al. A Review on Mangrove Actinobacterial Diversity: The Roles of Streptomyces and Novel Species Discovery. Prog Microbes Mol Biol 2019; 2(1): a00000024.

11. Lee L-H, Chan K-G, Stach J, et al. The search for biological active agent (s) from actinobacteria. Front Microbiol 2018; 9: 824

12. Ser H-L, Ab Mutalib NS, Yin W-F, et al. Evaluation of antioxidative and cytotoxic activities of Streptomyces pluripotens MUSC 137 isolated from mangrove soil in Malaysia. Front Microbiol 2015; 6: 1398

13. Ser H-L, Palanisamy UD, Yin W-F, et al. Streptomyces malaysiense sp. nov: a novel Malaysian mangrove soil actinobacterium with antioxidative activity and cytotoxic potential against human cancer cell lines. Sci Rep 2016; 6(1): 1-12.

14. Law JW-F, Letchumanan V, Tan LT-H, et al. The Rising of "Modern Actinobacteria" Era. Prog Microbes Mol Biol 2020; 3(1): a00000064.

15. Tan LT-H, Ser H-L, Yin W-F, et al. Investigation of antioxidative and anticancer potentials of Streptomyces sp. MUM256 isolated from Malaysia mangrove soil. Front Microbiol 2015; 6: 1316.

16. Law JW-F, Ser H-L, Duangjai A, et al. Streptomyces colonosanans sp. nov., a novel actinobacterium isolated from Malaysia mangrove soil exhibiting antioxidative activity and cytotoxic potential against human colon cancer cell lines. Front Microbiol 2017; 8: 877.

17. Tan LT-H, Chan K-G, Pusparajah P, et al. Mangrove derived Streptomyces sp. MUM265 as a potential source of antioxidant and anticolon-cancer agents. BMC Microbiol 2019; 19(1): 38.

18. Lee L-H, Ser H-L, Ab Mutalib NS, et al. Winning the war against colon cancer: chemo-preventive potential of novel Streptomyces specie derived from mangrove forest in Malaysia. GUT 2018; 67: A13-14.

19. Lee L-H, Law JW-F, Khan TM, et al. Unveiling the anti-colon cancer potential of Sarawak mangrove-derived novel Streptomycetes. GUT 2019; 68: A42-43

20. Eng SK, Tan LTH, Goh BH, et al. KRAS as potential target in colorectal cancer therapy. In: Natural Bio-active Compounds, Volume 1: Production and Applications. Singapore: Springer; 2019. p. 389-424.

21. Arrington AK, Heinrich EL, Lee W, et al. Prognostic and predictive roles of KRAS mutation in colorectal cancer. Int J Mol Sci 2012; 13: 12153-12168.

22. Andreyev HJ, Norman AR, Cunningham D, et al. Kirsten ras mutations in patients with colorectal cancer: The multicenter "RASCAL" study. J Natl Cancer Inst 1998; 90: 675-684.

23. Kopreski MS, Benko FA, Kwee C, et al. Detection of mutant K-ras DNA 
in plasma or serum of patients with colorectal cancer. Br J Cancer 1997; 76: $1293-1299$

24. Bell SM, Scott N, Cross D, et al. Prognostic value of p53 overexpression and c-Ki-ras gene mutations in colorectal cancer. Gastroenterol 1993; 104: $57-64$

25. Bishehsari F, Mahdavinia M, Malekzadeh M, et al. Patterns of K-ras mutation in colorectal carcinomas from Iran and Italy (a Gruppo Oncologico dell'Italia Meridionale study): influence of microsatellite instability status and country of origin. Ann Oncol 2006; 17(7): 91-96.

26. Chan AO, Soliman AS, Zhang Q, et al. Differing DNA methylation patterns and gene mutation frequencies in colorectal carcinomas from middle eastern countries. Clin Cancer Res 2005; 11: 8281-8287.

27. Wu CM, Tang R, Wang JY, et al. Frequency and spectrum of K-RAS codons 12 and 13 mutations in colorectal adenocarcinomas from Taiwan. Cancer Genet Cytogenet 2005; 158(1): 55-60.

28. Samowitz WS, Curtin K, Schaffer D, et al. Relationship of Ki-ras mutations in colon cancers to tumor location stage and survival: a population-based study. Cancer Epidemiol Biomarkers Prev 2000; 9: 1193-1197.

29. Bazan V, Migliavacca M, Zanna I, et al. Specific codon 13 K-ras mutations are predictive of clinical outcome in colorectal cancer patients, whereas codon $12 \mathrm{~K}$-ras mutations are associated with mucinous histotype. Ann Oncol 2002; 13: 1438-1446.

30. Bos JL, Fearon ER, Hamilton SR, et al. Prevalence of ras gene mutations in human colorectal cancers. Nature 1987; 327: 293-297.

31. Forrester K, Almoguera C, Han K, et al. Detection of high incidence of K-ras oncogenes during human colon tumorigenesis. Nature 1987; 327: 298-303.

32. Reinacher-Schick A, Schulmann K, Modest D, et al. Effect of KRAS codon 13 mutations in patients with advanced colorectal cancer (advanced CRC) under oxaliplatin containing chemotherapy. Results from a translational study of the AIO colorectal study group. BMC Cancer 2012; 12(349).

33. Lievre A, Bachet J, Le Corre D, et al. KRAS mutation status is predictive of response to cetuximab therapy in colorectal cancer. Cancer Res 2006; 66: 3992-3995.

34. Ambros V. The functions of animals microRNAs. Nature 2004; 431: 350-355.

35. Harfe B. MicroRNAs in vertebrate development. Curr Opin Genet Dev 2005; 15: 410-415.

36. Bartel DP. MicroRNAs: genomics, biogenesis, mechanism and function. Cell 2004; 116: 281-297.

37. Lee RC, Feinbaum RL and Ambros V. The C. elegans heterochronic gene lin-4 encodes small RNAs with antisense complementarity to lin14. Cell 1993; 75(5): 843-854.

38. Almeida MI, Reis RM and Calin GA. MicroRNA history: Discovery, recent applications, and next frontiers. Mutat Res 2011; 711: 1-8

39. Reinhart BJ, Slack FJ, Basson M, et al. The 21-nucleotide let-7 RNA regulates developmental timing in Caenorhabditis elegans. Nature 2000; 403(6772): 901-906.

40. Pasquinelli AE, Reinhart BJ, Slack F, et al. Conservation of the sequence and temporal expression of let-7 heterochronic regulatory RNA. Nature 2000; 408(6808): 86-89.

41. Calin GA and Groce CM. MicroRNA signatures in human cancers. Natl Rev Cancer 2006; 6(11): 857-866.

42. Cullen BR. Transcription and processing of human microRNA precursors. Mol Cell 2004; 16(6): 861-865.

43. Van Rooij E, Sutherland LB, Liu N, et al. A signature pattern of stressresponsive microRNAs that can evoke cardiac hyperthrophy and heart failure. Proc Natl Acad Sci U S A 2006; 103(48): 18255-18260.

44. Tijsen AJ, Creemers EE, Moerland PD, et al. MiR-423-5p as a circulating biomarker for heart failure. Circ Res 2010; 106: 1035-1039.

45. Corsten MF, Dennert R, Jochems S, et al. Circulating MicroRNA$208 \mathrm{~b}$ and MicroRNA-499 reflect myocardial damage in cardiovascular disease. Circ Cardiovasc Genet 2010; 3: 499-506.

46. Stanczyk J, Pedrioli DM, Brentano F, et al. Altered expression of MicroRNA in synovial fibroblasts and synovial tissue in rheumatoid arthritis. Arthritis Rheum 2008; 58(4): 1001-1009.

47. Nakasa T, Miyaki S, Okubo A, et al. Expression of microRNA-146 in rheumatoid arthritis synovial tissue. Arthritis Rheum 2008; 58(5): $1284-1292$.

48. Dai Y, Huang YS, Tang M, et al. Microarray analysis of microRNA expression in peripheral blood cells of systemic lupus erythematosus patients. Lupus 2007; 16(12): 939-946.

49. Pan W, Zhu S, Yuan M, et al. MicroRNA-21 and microRNA-148a contribute to DNA hypomethylation in lupus CD4+ T cells by directly and indirectly targeting DNA methyltransferase 1. J Immunol Res 2010; 184(12): 6773-6781.

50. Lukiw WJ. Micro-RNA speciation in fetal, adult and Alzheimer's disease hippocampus. Neuroreport 2007; 18(3): 297-300.

51. Lukiw WJ and Pogue AI. Induction of specific micro RNA (miRNA) species by ROS-generating metal sulfates in primary human brain cells. J Inorg Biochem 2007; 101:1265-1269.

52. Kim J, Inoue K, Ishii J, et al. A microRNA feedback circuit in midbrain dopamine neurons. Sci 2007; 317(5842): 1220-1224.

53. Calin GA, Dumitru CD, Shimizu M, et al. Frequent deletions and down-regulation of micro-RNA genes miR15 and miR16 at 13q14 in chronic lymphocytic leukemia. Proc Natl Acad Sci U S A 2002; 99(24): 15524-15529.

54. Cimmino A, Calin GA, Fabbri M. miR-15 and miR-16 induce apoptosis by targeting BCL2. Proc Natl Acad Sci U S A 2005; 102(39): 13944 13949 .

55. Ab Mutalib NS, Cheah Y-K, Abdul Rahman S, et al. Differential microRNA expression and identification of putative miRNA targets and pathways in head and neck cancer. Int J Mol Med 2011; 28:327-336.
56. Ab Mutalib NS, Lee L-H, Mohd Sidik S, et al. miR-181a regulates multiple pathways in hypopharyngeal squamous cell carcinoma. Afr J Biotechnol 2012; 11(22): 6129-6137.

57. Ab Mutalib NS, Lee L-H and Cheah Y-K. miR-205 in situ expression and localization of head and neck tumor tissue array. Asian Pac J Cancer Prev 2014; 15 (21): 9071-9015.

58. Sulaiman SA, Ab Mutalib NS and Jamal R. miR-200c Regulation of Metastases in Ovarian Cancer: Potential Role in Epithelial and Mesenchymal Transition. Front Pharmacol 2016; 7:271.

59. Johnson SM, Grosshans H, Shingara J, et al. RAS is regulated by the let-7 microRNA family. Cell 2005; 120(5): 635-647.

60. Garzon R, Volinia S, Liu CG, et al. MicroRNA signatures associated with cytogenetics and prognosis in acute myeloid leukemia. Blood 2008; 111: 3183-3189.

61. He L, He X, Lim LP, et al. A microRNA component of the p53 tumor suppressor network. Nature 2007; 447: 1130-1134.

62. Kluiver J, Poppema S, De Jong D, et al. BIC and miR-155 are highly expressed in Hodgkin, primary mediastinal and diffuse large B cell lymphomas. J Pathol 2005; 207(2): 243-249.

63. Metzler M, Wilda M, Busch K, et al. High expression of precursor microRNA-155/BIC RNA in children with Burkitt lymphoma. Genes Chromosomes Cancer 2004; 38(2): 167-169.

64. Volinia S, Calin GA, Liu C-G, et al. A microRNA expression signature in human solid tumors defines cancer targets. Proc Natl Acad Sci U S A 2006; 103: 2257-2261.

65. Dong $\mathrm{Y}, \mathrm{Wu} \mathrm{WKK}, \mathrm{Wu} \mathrm{CW}$, et al. MicroRNA dysregulation in colorectal cancer: A clinical perspective. Br J Cancer 2011; 104: 893-898.

66. Xi Y, Formentini A, Chien M, et al. Prognostic values of microRNAs in colorectal cancer. Biomark Insights 2006; 1: 113-121.

67. Hon KW, Abu N, Ab Mutalib N-S, et al. miRNAs and lncRNAs as Predictive Biomarkers of Response to FOLFOX Therapy in Colorectal Cancer. Front Pharmacol 2018; 9:846.

68. Faltejskova P, Besse A, Sevcikova S, et al. Clinical correlations of miR21 expression in colorectal cancer patients and effects of its inhibition on DLD1 colon cancer cells. Int J Colorectal Dis 2012; 27(11): 1401-1408.

69. Link A, Balaguer F, Shen Y, et al. Fecal microRNAs as novel biomarkers for colon cancer screening. Cancer Epidemiol Biomarkers Prev 2010; 19(7): 1766-1774

70. Kjaer-Frifeldt S, Hansen TF, Nielsen BS, et al. The prognostic importance of miR-21 in stage II colon cancer: A population-based study. Br J Cancer 2012; 107(7): 1169-1174.

71. Kanaan Z, Rai SN, Eichenberger MR, et al. Plasma miR-21: a potential diagnostic marker of colorectal cancer. Ann Surg 2012; 256(3): 544-551.

72. Catela IT, Aralica G, Cacev T, et al. miR-106a overexpression and pRB downregulation in sporadic colorectal cancer. Exp Mol Pathol 2012; 94(1): $148-154$

73. Feng B, Dong TT, Wang LL, et al. Colorectal cancer migration and invasion initiated by microRNA-106a. PloS One, 2012; 7(8): e43452.

74. Diaz R, Silva J, Garcia JM, et al. Deregulated expression of miR-106a predicts survival in human colon cancer patients. Genes Chromosomes Cancer 2008; 47(9): 794-802.

75. Faltejskova P, Svoboda M, Srutova K, et al. Identification and functional screening of microRNAs highly deregulated in colorectal cancer. J Cell Mol Med 2012; 16(11): 2655-2666.

76. Xu XM, Qian JC, Deng ZL, et al. Expression of miR-21, miR-31, miR96 and miR-135b is correlated with the clinical parameters of colorecta cancer. Oncol Lett 2012; 4(2): 339-345.

77. Nagel R, Le Sage C, Diosdado B, et al. Regulation of the adenomatous polyposis coli gene by the miR-135 family in colorectal cancer. Cancer Res 2008; 68(14): 5795-5802.

78. Sarver AL, French AJ, Borralho PM, et al. Human colon cancer profiles show differential microRNA expression depending on mismatch repair status and are characteristic of undifferentiated proliferative states. BMC Cancer 2009; 9(401).

79. Reid JF, Sokolova V, Zoni E, et al. miRNA profiling in colorectal cancer highlights miR-1 involvement in MET-dependent proliferation. Mol Cancer Res 2012; 10(4): 504-515.

80. Yang $\mathrm{M}-\mathrm{H}$, Lin B-R, Chang $\mathrm{C}-\mathrm{H}$, et al. Connective tissue growth factor modulates oral squamous cell carcinoma invasion by activating a miR-504/ FOXP1 signalling. Oncogene 2012; 31: 2401-2411.

81. Hu W, Chan CS, Wu R, et al. Negative regulation of tumor suppressor p53 by microRNA miR-504. Mol Cell 2010; 38(5): 689-699.

82. Feng Z, Zhang C, Wu R, et al. Tumor suppressor p53 meets microRNAs. J Mol Cell Biol 2011; 3(1): 44-50.

83. Bauer KM and Hummon AB. Effects of the miR-143/-145 microRNA cluster on the colon cancer proteome and transcriptome. J Proteome Res 2012; 11(9): 4744-4754.

84. Kent OA, Chivukula RR, Mullendore M, et al. Repression of the miR$143 / 145$ cluster by oncogenic Ras initiates a tumor-promoting feed-forward pathway. Genes Dev, 2010; 24: 2754-2759.

85. Liu R, Liao J, Yang M, et al. The cluster of miR-143 and miR-145 affect the risk for esophageal squamous cell carcinoma through co-regulating Fascin Homolog 1. PloS One, 2012; 7(3): e33987

86. Chiyomaru T, Enokida H, Tatarano S, et al. miR-145 and miR-133a function as tumour suppressor and directly regulate FSCN1 expression in bladder cancer. Br J Cancer 2010; 102(5): 883-891.

87. Cekaite L, Rantala JK, Bruun J, et al. MiR-9, -31 and -182 deregulation promote proliferation and tumor cell survival in colon cancer. Neoplasia 2012; 14(9): 868-879.

88. Hamfjord J, Stangeland AM, Hughes T, et al. Differential expression of miRNAs in colorectal cancer: comparison of paired tumor tissue and adjacent normal mucosa using high-throughput sequencing. PloS One, 2012; 7(4): e314150

89. Yu S, Lu Z, Liu C, et al. miRNA-96 suppresses KRAS and function as a tumor suppressor gene in pancreatic cancer. Cancer Res, 2010; 70(14): 
6015-6025

90. Sarver AL, Li L and Subramanian S. MicroRNA miR-183 function as an oncogene by targeting the transcription factor EGR1 and promoting tumor cell migration. Cancer Res, 2010; 70(23): 9570-9580.

91. Arndt GM, Dossey L, Cullen LM, et al. Characterization of global microRNA expression reveals oncogenic potential of miR-145 in metastatic colorectal cancer. BMC Cancer 2009; 9(374).

92. Mencia N, Selga E, Noe V, et al. Underexpression of miR-224 in methotrexate resistant human colon cancer cells. Biochem Pharmacol 2011; 82(11): 1572-1582.

93. Luo X, Burwinkel B, Tao S, et al. MicroRNA signatures: novel biomarker for colorectal cancer? Cancer Epidemiol Biomarkers Prev 2011; 20(7): 1272-1286

94. Chiang Y, Song Y, Wang Z, et al. Aberrant expression of miR-203 and its clinical significance in gastric and colorectal cancers. J Gastrointest Surg 2011; 15(1): 63-70.

95. Schee K, Boyce $\mathrm{K}$, Abrahamsen TW, et al. Clinical relevance of microRNA miR-21, miR-31, miR-92a, miR-101, miR-106a and miR145 in colorectal cancer. BMC Cancer 2012; 12(505).

96. Wang CJ, Zhou ZG, Wang L, et al. Clinicopathological significance of microRNA-31, -143 and -145 expression in colorectal cancer. Dis Markers 2009; 26(1): 27-34.

97. Luo H, Zou J, Dong Z, et al. Up-regulated miR-17 promotes cell proliferation, tumour growth and cell cycle progression by targeting the RND3 tumour suppressor gene in colorectal carcinoma. Biochem J 2012; 442: 311-321.

98. Slaby O, Svoboda M, Michalek J, et al. MicroRNAs in colorectal cancer: translation of molecular biology into clinical application. Mol Cancer 2009; 8(102): 1-13

99. Ciardiello F and Tortora G. EGFR antagonists in cancer treatment. N Engl J Med 2008; 358: 1160-1174.

100. Vogelstein B, Fearon ER, Hamilton SR, et al. Genetic alterations during colorectal-tumor development. N Engl J Med 1988; 319 525-532.

101. Meng F, Henseon R, Wehbe-Janek H, et al. MicroRNA-21 regulates expression of the PTEN tumor suppressor gene in human hepatocellular cancer. Gastroenterol 2007; 133: 647-658.

102. Fearon ER and Vogelstein B. A genetic model for colorectal tumorigenesis. Cell 1990; 61: 759-767.

103. Cheah Y-K, AB Mutalib NS, Mohd Sidik S, et al. P53 codon 73 polymorphisms and random amplified polymorphic DNA analysis of non-melanoma skin cancer through archival formalin-fixed paraffinembedded tissue. Oncol Rep 2012; 27:753-763.

104. Zhu S, Wu H, Wu F, et al. MicroRNA-21 targets tumor suppressor genes in invasion and metastasis. Cell Res 2008; 18: 350-359.

105. Schimanski CC, Frerichs K, Rahman F, et al. High miR-196a levels promote the oncogenic phenotype of colorectal cancer cells. World J Gastroenterol 2009; 15(17): 2089-2096.

106. Rossi S, Kopetz S, Davuluri R, et al. MicroRNAs, ultraconserved genes and colorectal cancers. Int J Biochem Cell Biol 2010; 42(8): 1291-1297.

107. Kim S, Choi M, and Cho K-H. Identifying the target microRNAs in colorectal cancer. Comput Biol Chem 2009; 33: 94-99. 